\title{
Advantages and Pitfalls of Three-Dimensional Ultrasound Imaging of Carotid Bifurcation
}

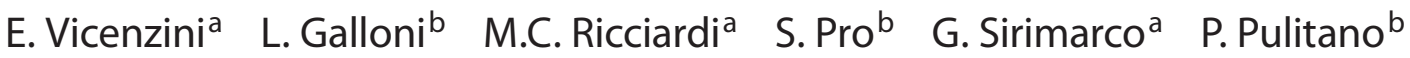 \\ O. Mecarellib $^{b}$ V.Di Piero ${ }^{a} \quad$ G.L. Lenzi ${ }^{a}$ \\ ${ }^{\text {a Stroke Unit and }}{ }^{\mathrm{b}}$ Neurophysiology Unit, Department of Neurology and Psychiatry, Sapienza University of Rome, \\ Rome, Italy
}

\section{Key Words}

Three-dimensional ultrasound - Carotid artery imaging • Extracranial carotid artery tortuosity and kinking $\cdot$ Carotid stenosis

\begin{abstract}
Objectives: Several specialists use three-dimensional (3D) ultrasound as adjuvant imaging technique in their clinical practice. It has been applied to study carotid plaque morphology, surface and volume during atherosclerosis progression. Nonetheless, no papers have so far described the use of this technique in conditions different than carotid stenosis, such as bifurcation anatomy changes of the caliber and vessel course modifications. Methods: Patients admitted to our ultrasound laboratory for vascular screening were submitted to standard carotid duplex and to 3D ultrasound reconstruction of the carotid bifurcation. Results: Forty normal subjects, 7 patients with caliber alterations (4 carotid bulb ectasia and 3 internal carotid lumen narrowing), 45 patients with course variations (tortuosities and kinking) and 35 patients with internal carotid artery stenosis of various degrees have been investigated. Conclusions: 3D ultrasound is a feasible technique. It can improve carotid axis imaging through a better presentation of caliber variations and vessel course 'at a glance'. 3D ultrasound from the inward
\end{abstract}

flow can provide imaging of the stenosis, but stenosis quantification should always take into account the assessment of plaque morphology and vessel wall.

Copyright $\odot 2011$ S. Karger AG, Basel

\section{Introduction}

The possibility of visualizing internal organs threedimensionally has always been fascinating in the field of medical imaging, for the theoretical possibility of obtaining a virtual inner visualization, simulating reality. The virtual three-dimensional (3D) image presentation may be useful for surgeons, for example, to better study anatomical boundaries of the structures to be submitted to surgical procedures. When data digitalization became standard in medical imaging, the offline-computed enhancement allowed the development of several pieces of postprocessing software for reconstructing the acquired images in order to visualize planes different from the one used during acquisition and, hence, for 3D imaging and navigation.

Ultrasound (US) is a very diffuse, relatively low-cost, noninvasive imaging technique and, nowadays, also used with very versatile pieces of software for digital 3D postprocessing imaging. Several specialists indeed benefit

Edoardo Vicenzini, MD, PhD

Department of Neurology and Psychiatry, Sapienza University of Rome

Viale dell'Università 30

IT-00185 Rome (Italy)

Tel. +39 064991 4705, E-Mail edoardo.vicenzini@uniromal.it 
from 3D US: cardiologists use it as a valid corroborative diagnostic tool to standard two-dimensional imaging for cardiac diseases [1-5], obstetrics and gynecologists use it for fetal prenatal diagnosis $[6,7]$ and uterine and ovarian evaluation $[8,9]$, surgeons for breast cancer [10] and gastroenterologists for focal liver lesions [11]. Moreover, as US is a real-time imaging technique, it was possible to add 'movement' as the 4th dimension (4D), developing 4D US, with impressive results in cardiac and fetal prenatal imaging $[12,13]$. All these 3D/4D modalities have the advantage of producing comprehensible US images also to the nonexperts, and they even exert positive effects on maternal emotional status during pregnancy [14].

Vascular surgeons can also benefit from 3D vessel reconstruction, usually achieved with CT and MRI scans [15], but also with US when vascular districts are accessible to evaluation [16]. Carotid bifurcation can be studied using both 3D imaging with CT and MRA [17], as well as US reconstructions [18-24]. Several authors described the possibility of monitoring carotid plaque volume or surface with 3D US in the presurgical evaluation [25], in the progression of atherosclerosis [26-28] or to evaluate the effects of therapies aimed at plaque remodeling [29]. Fundamentally, this approach semiautomatically reconstructs plaque volume and surface, identifying and presenting the atherosclerotic lesion according to the borders, indentified in B-mode and with the aid of the color, tracing its contours in different adjacent slices.

The 3D reconstruction of the carotid bifurcation from its inward flow is possible with US [30], but no papers have so far described the use of this technique in conditions different than carotid stenosis, such as bifurcation changes of the caliber and vessel course alterations. The aim of this review was to explore the possibility of visualizing the carotid bifurcation with 3D US reconstructions from the inward flow in several conditions: in normal subjects, in subjects with caliber alterations (carotid bulb ectasia and internal carotid artery caliber narrowing) and vessel course modifications (tortuosities and kinking). Further, patients with internal carotid stenosis of different degrees were also evaluated. Possibilities, advantages and disadvantages of the technique are discussed.

\section{Subjects and Methods}

A series of patients admitted to the Neurosonology Laboratory of the Department of Neurological Sciences for vascular screening have been examined with both standard duplex and 3D
US imaging in the same session. Patients were selected among those presenting a better neck echogenicity in order to obtain better images. Forty normal subjects, 7 patients with caliber alterations ( 4 with carotid bulb ectasia and 3 with internal carotid lumen narrowing), 45 patients with course variations (tortuosities and kinking) and 35 patients with internal carotid artery stenosis of various degrees have been investigated. The 3D US imaging was part of the routine evaluation and patients gave their written informed consent.

Technical Procedure, Echographic Apparatus and 3D Imaging

A Siemens S2000 US echograph with high-frequency linear probes $(9,14$ and $18 \mathrm{MHz}$ ) was used for standard imaging of the carotid bifurcation and for the $3 \mathrm{D}$ reconstruction. The proprietary 3D/4D reconstruction software from the Siemens S2000 system (rev 1.6) was used directly on the machine.

Standard two-dimensional carotid bifurcation imaging, in longitudinal and axial planes anteriorly or posteriorly to the sternocleidomastoideus muscle, was used with B-mode, color/power imaging and triplex scan with pulsed-wave Doppler, in order to detect carotid bifurcation anatomy, vessel course variations, flow turbulences and the presence of carotid stenosis, as described elsewhere $[31,32]$.

Immediately after the standard two-dimensional imaging, the $3 \mathrm{D}$ volume scans were recorded manually. After fixing the proximal tract of the common carotid artery in the center of the display in the transversal plane, a test axial scanning was performed from the proximal carotid artery to the distal internal carotid artery at approximately $1 \mathrm{~cm}$ per second - to adjust the visualization. The 3D US software was then switched on to record the volume scan: the power box was set to the orthogonal $90^{\circ}$ angle position; pulse repetition frequency, color gain and color persistence were adjusted during a second test axial scan in order to reduce artifacts due to the inward flow color signal overlapping the vessel wall and to minimize color 'flashing' due to the blood pulsatility. The software features 'axial reconstruction' and 'medium resolution' - that is set for a length of $10 \mathrm{~cm}$ to be scanned in $12 \mathrm{~s}$ - were selected. Data acquisition was then started and stopped manually; a bar control on the screen displayed the feedback for maintaining a constant straight direction and scan velocity. At the end of the scan, the 3D US 'volume rendering' reconstruction of the acquired volume set was started on the system. After the global 3D image presentation, B-mode imaging was excluded and color magnification (color priority) adjusted to optimize the final visualization of the vessels. When necessary, signals from unnecessary structures, such as the internal jugular vein and/or external artifacts, were deleted from the 3D reconstruction.

Fixed images (DICOM format) and small longitudinal or axial rotational $\left(15-30^{\circ}\right)$ videos of the $3 \mathrm{D}$ reconstruction (DICOM format) were exported to the PC using the Showcase 5.0 software (Trilltech Inch) for offline analysis. The files were converted: images into .jpg and videos into avi formats. When images were not satisfactory after the first 3D volume acquisition, a second 3D scan and volume rendering was performed again on the US machine, to achieve better images, and then exported to the PC.

Normal Visualization

3D US reconstruction in normal subjects allows a good visualization of the carotid bifurcation. In figure 1, two examples are presented: all extracranial carotid arteries are easily identifiable. 
Fig. 1. 3D US reconstruction of normal bifurcation. IC = Internal carotid artery; $\mathrm{EC}=$ external carotid artery; BLB = carotid bulb; $\mathrm{CC}=$ common carotid artery. The arrow indicates the superior thyroid artery. On the right sight of the figure, the three-plane reconstruction of the axial 3D scanning generated by the software is depicted.

Fig. 2. Duplex scan of carotid bulb ecasia. a B-mode. b Power. c Color. Note the vessel dilatation in the two-dimensional images and the flow turbulences in the color images in c. 3D US reconstruction (d) shows the bulb ectasia with its anatomical boundaries.
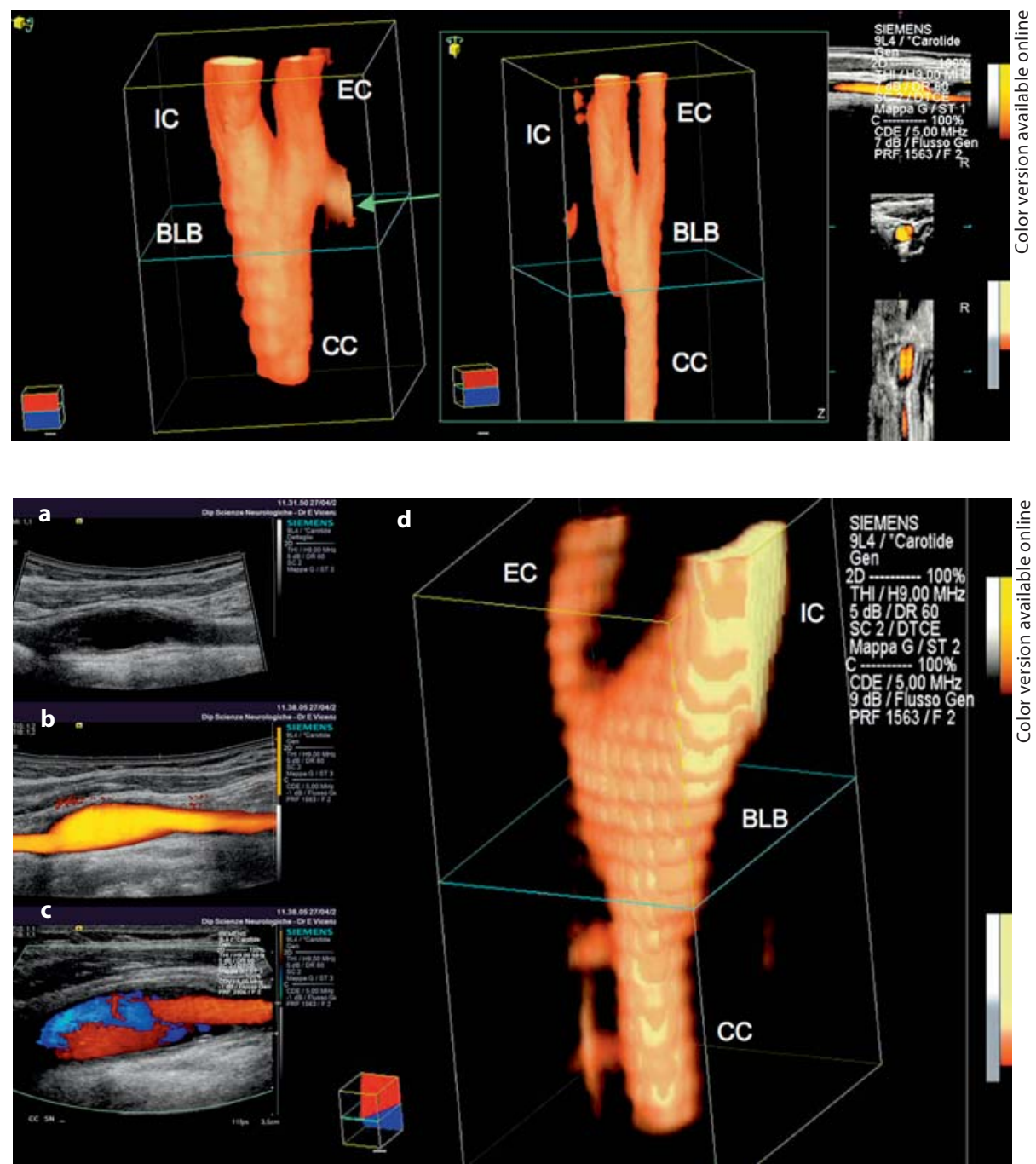

The $3 \mathrm{D}$ reconstruction software also allows the 3-plane two-dimensional visualization (B-mode, power mode, isolated or together) with easy navigation through the different planes (fig. 1, right side). Small rotational videos (online suppl. video 1, www.karger. com/doi/10.1159/000327694) help to better visualize the vessels from different angles. Depending on the breath inspiration/expiration phase, the internal jugular vein may also be detectable. However, during image postprocessing, the jugular vein image can be deleted, in order to better focus the attention on the arterial axis.

\section{Caliber Alterations}

Carotid Bulb Ectasia

The carotid bulb dilatation is characterized by a fusiform enlargement (usually 1-1.5 cm of anteroposterior diameter), usually involving the distal tract of the common carotid artery and extending through the bulb to the internal carotid artery origin. Frequently, it is observed in hypertensive aged patients and in Takayasu arteritis, provoking flow turbulences [33].

Two-dimensional images in B-mode (fig. 2a), power (fig. 2b) and color modes (fig. 2c) can easily detect the vessel enlargement, also allowing precise vessel diameter measurements. When the dilatation is important, flow turbulences can be detected in the color mode, as a red/blue pattern and with 'aliasing' effect (fig. 2c; online suppl. video 2). The 3D reconstruction, rotating in the different planes allows a better global identification of the anatomy of the ectasia (fig. 2d; online suppl. video 3). However, the reconstruction images always have to be considered with caution for final diagnostic decisions, as flow disturbances can cause several artifacts in the postprocessing reconstruction: as for every $3 \mathrm{D}$ reconstruction in vascular imaging in general, final $3 \mathrm{D}$ pictures cannot be considered alone and without the previous or concomitant mandatory analysis of the two-dimensional images.

Internal Carotid Artery Caliber Alterations

In particular cases, 3D imaging of the internal carotid artery may be helpful for the visualization of the vessel patency. The hemodynamic evaluation must always be considered as fundamental to decide whether a vessel is occluded or not (fig. 3a, b), but the 3D reconstruction (fig. 3c; online suppl. video 4) can easily demonstrate the internal carotid artery patency, adding the 


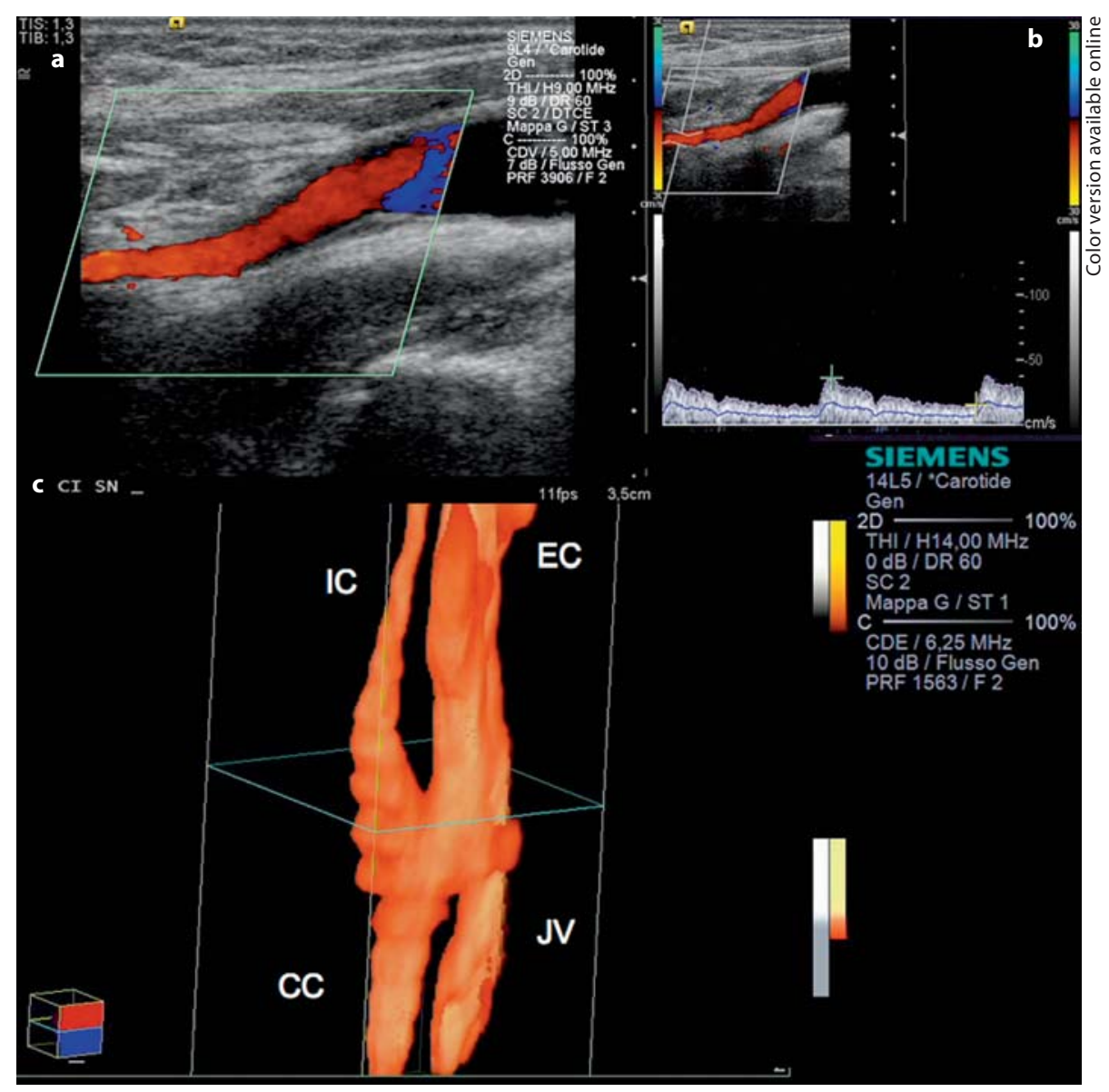

Fig. 3. Duplex scan of distal internal carotid artery in a case of internal carotid recanalization after occlusion provoked by dissection. a Color. b Pulsed-wave Doppler. 3D US reconstruction (c) shows the narrowed lumen of the recanalized internal carotid artery, corresponding to the low blood flow velocities in $\mathbf{b}$.

images for interpretation. The case presented in figure 3 is a subject with distal internal carotid artery recanalization after dissection [34]. Note the reduced blood flow velocities in the distal internal carotid artery (fig. 3b) and the narrowed distal vessel lumen in the $3 \mathrm{D}$ images. Moreover, in the $3 \mathrm{D}$ set, note the anatomical boundaries and the internal jugular vein (fig. $3 \mathrm{c}$; online suppl. video 4)

\section{Internal Carotid Artery Course Variations (Tortuosities and Kinking)}

Extracranial vessel course abnormalities are generally asymptomatic and can be observed very often in the general population $[35,36]$. These can be classified as 'tortuosities' and 'kinking', when changes in the vessel course are greater than $90^{\circ}$. Usually, tortuosities and kinking are observed in the $4-\mathrm{cm}$ extracranial segment of the internal carotid artery, more often in the distal tract of the vessel, in females (M/F: 1/4), congenital or secondary to aging and atherosclerosis processes and consequent to fibromuscular hyperplasia [37-39]. Even though these alterations are asymptomatic and without clinical relevance in the normal subject, tortuosities and kinking have to be identified prior to surgical procedures, since they may hinder - for example - the intravascular positioning of a stent, while the anatomical approach and clamping of the internal carotid artery may be easier during endarterectomy [40].
Two-dimensional standard duplex can display course abnormalities as an abnormal curvature of the vessels that, in the color mode, also show the changes of the blood flow direction according to the vessel direction change. The power Doppler, with an orthogonal insonation angle, can be of help for the better visualization of the whole vessel course, especially where the color mode fails to depict the vessels in tortuous sections. Moreover, it has to be kept in mind that artery course variations can induce changes in hemodynamics - with blood flow velocity reduction - in the segment proximal to the tortuosity, which may then be erroneously misinterpreted as a distal vessel occlusion, if the distal kinking is not recognized. While in the two-dimensional images it is usually necessary to repeatedly correct the color box insonation angle or to adjust the probe orientation to obtain optimal complete vessel recognition, the $3 \mathrm{D}$ reconstruction can be of help to gain the whole visualization 'at a glance'. Figure 4 presents three examples: a distal internal carotid artery kinking (fig. 4a), a tortuous course of the internal carotid artery origin with intermediate kinking (fig. 4b) and a distal tortuosity of the internal carotid artery (fig. 4c). Note the changes of the blood flow direction according to the vessel course variations in the color modes (fig. $4 \mathrm{a}, \mathrm{c}$, boxes $\mathrm{A}$ ) and the better visualization in the power modes (fig. 4 , boxes $\mathrm{B}$ ). The $3 \mathrm{D}$ reconstructions (fig. 4 , boxes $\mathrm{C}$ ) give a better complete representation of the vessel course modification, and even better while rotating the videos (online suppl. videos 5-7). 


\section{Internal Carotid Artery Stenosis}

According to data published with 3D US on plaque volume reconstruction and from the experience with other neuroimaging conventional techniques, such as CT and MRA angiography, internal carotid artery stenosis has been analyzed with $3 \mathrm{D}$ reconstructions according to 2 principles: (1) by $3 \mathrm{D}$ reconstruction of the internal carotid plaque structure from either the US Bmode and/or from the vessel wall parenchymal (CT/MRI) imaging; (2) by $3 \mathrm{D}$ reconstruction of the residual lumen, visualized with the power Doppler or with other imaging techniques [1724]. According to point 2, from the historical experience with conventional angiography, when 3D imaging was not yet diffuse and the vessel wall was not visible, the degree of the internal carotid artery stenosis was calculated as the percentage ratio between the residual lumen diameters in the highest narrowed point and the supposed-to-be-normal distal lumen (NASCET distal stenosis ratio) [41]. When imaging of the arterial wall has become standard, the 'local' criterion for stenosis evaluation was adopted, calculated as the ratio between the residual lumen diameters in the highest narrowed point and the whole external anatomical area of the vessel wall in the same segment of the stenosis (ECST) [42]. These two methods have their disadvantages, fundamentally represented by the possibility of underinterpretation of stenosis in case 1 , because the vessel considered as normal reference is - actually - only supposed to be so, and the vessel wall is not directly visualized. Nonetheless, in cases of highgrade stenoses, the hemodynamic effect with increased blood flow velocities could induce the visualization of the residual lumen to appear better than real, therefore underestimating the stenosis degree.

Figure 5a and $\mathrm{b}$ shows the power Doppler (top) and 3D US reconstructions (below) in two cases of moderate internal carotid artery stenosis. Note that the visualization of the 'missing part' of the vessel lumen in 3D US, reconstructed on the basis of the residual flow, is strictly correlated with the angle of the visualization (online suppl. videos 8,9 ). Figure 5 c shows an example of underestimation of a severe internal carotid artery stenosis with hemodynamic effect, visible with the 'aliasing' in the color images (fig. 5c top; online suppl. video 10). Increased blood flow velocities in this case induce a clear underestimation of the stenosis in the 3D US reconstruction (fig. 5c below; online suppl. video 11), because the image is computed on the base of the flow signal - increased in this case - from the inward flow. In these cases, the evaluation of the degree of stenosis must include the study of the vessel wall and cannot be excluded, also for its importance in analyzing plaque morphology.

In this study, only the $3 \mathrm{D}$ reconstruction of the residual lumen detected with the power mode was applied. However, this method is limited, similarly to all the techniques that base the imaging only on the inward flow: as a matter of fact, if the plaque is not considered, stenosis degree quantification is based on the comparison of what we only suppose to be normal, and hence is underestimated. Moreover, in these cases of the 3D US reconstruction, the blood flow pulsating at each cardiac cycle or the acoustic shadow of calcified plaques may create further artifacts: even if the persistence color setting is set to maximal values, blood flow slowing or stopping during diastole - especially in cases of very high resistive patterns as in the external carotid artery - induce the reduction or absence of signal, an artifact difficult to be eliminated even performing the scan as slow as
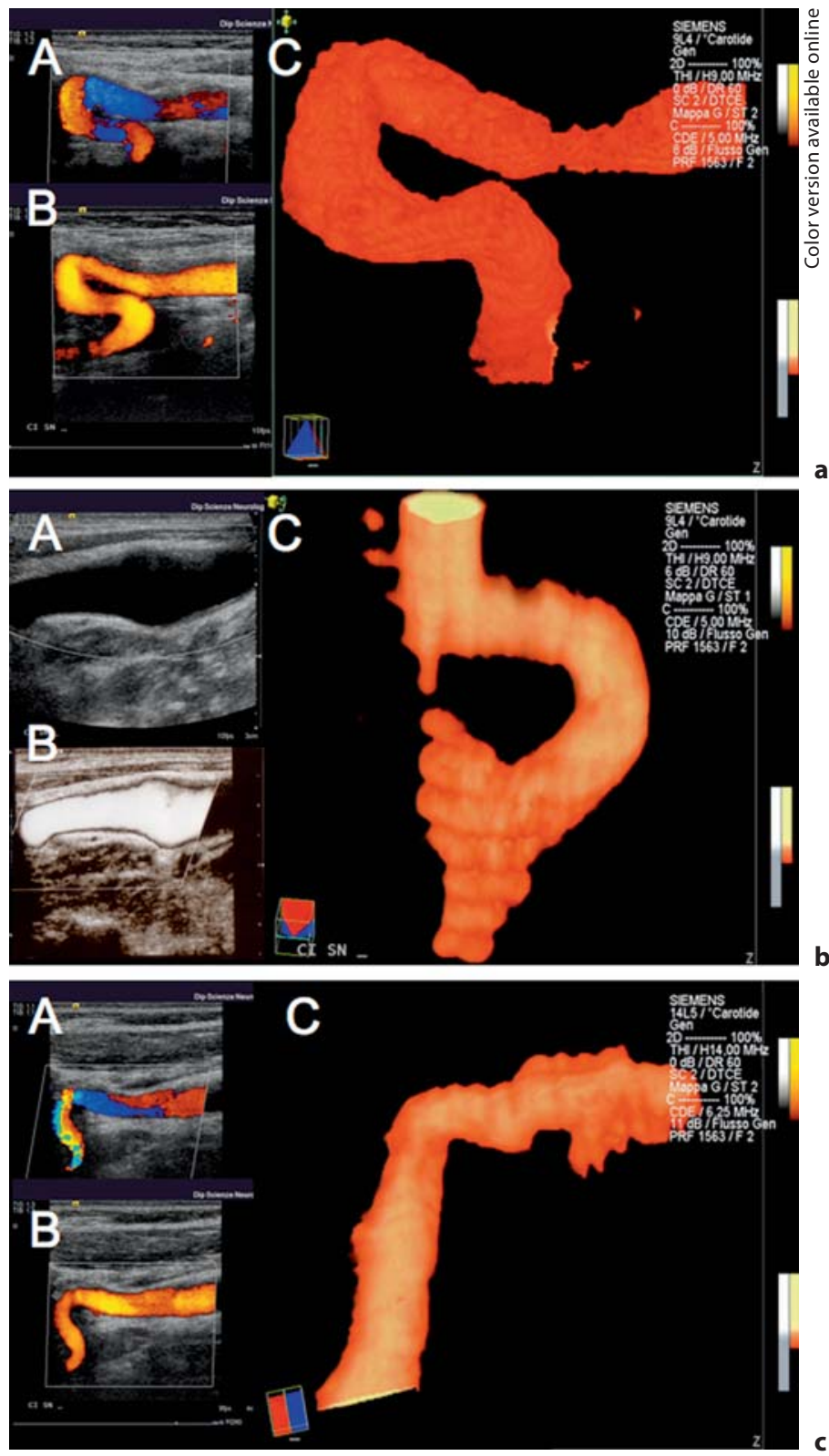

Fig. 4. Duplex scan (A, B) of distal internal carotid artery kinking (a), intermediated kinking with tortuous origin (b) and distal tortuosity (c). Note the color changes according to the vessel direction in A and the better vessel visualization with the power Doppler (B). The 3D US reconstruction (C) shows a better visualization 'at a glance' of the whole vessel course modification.

possible (fig. 6a, arrow). For the same reasons, the visualization of extracranial vessel occlusion with 3D US yields even less sensitivity, since the nonvisualization of the vessel is only the expression of absence of flow within the vessel, without considering what could have happened to the vessel wall (fig. 6b, dotted lines). 


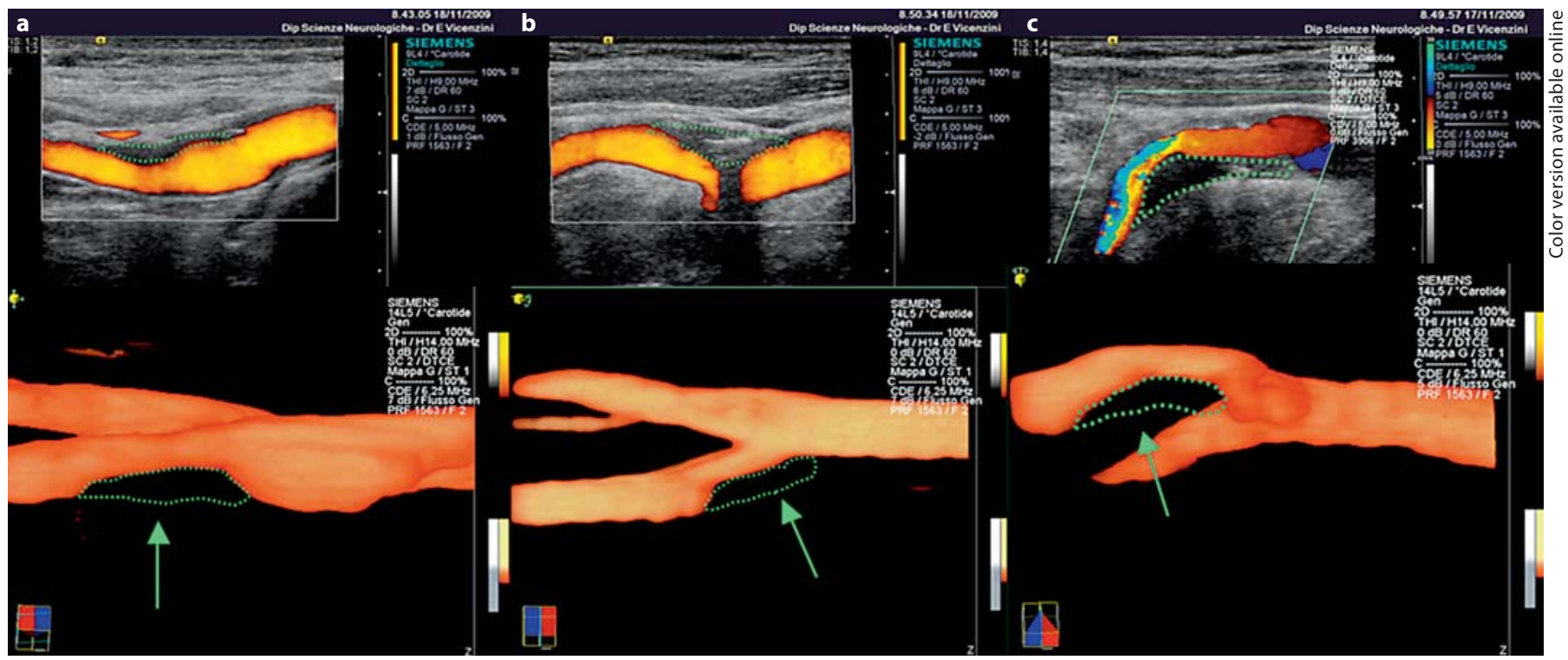

Fig. 5. Duplex scan of moderate, nonhemodynamic internal carotid stenosis (top $\mathbf{a}, \mathbf{b}$ ) with $3 \mathrm{D}$ reconstruction (bottom $\mathbf{a}, \mathbf{b}$ ). In case of severe, hemodynamic internal carotid artery stenosis (c), the hemodynamic effect causes flow turbulences and aliasing ef- fect at the color (top), with underestimation of the degree of stenosis at the 3D US reconstruction (bottom). Dotted lines and arrows indicate the extension of the plaque, designed according to two-dimensional imaging.

Fig. 6. a Typical artifact in 3D US reconstruction (arrows) in case of low diastolic flow due to a high-resistive pattern in the external carotid artery. The absence of signal may be erroneously interpreted as a vessel occlusion. Dotted lines indicate a 'void' area corresponding to internal carotid artery stenosis. b Internal carotid occlusion: in 3D images, the internal carotid artery cannot be visualized (dotted lines). Note that the artifacts in the bulb (arrows) due to the proximal resistive indices increase, secondarily to the occlusion.

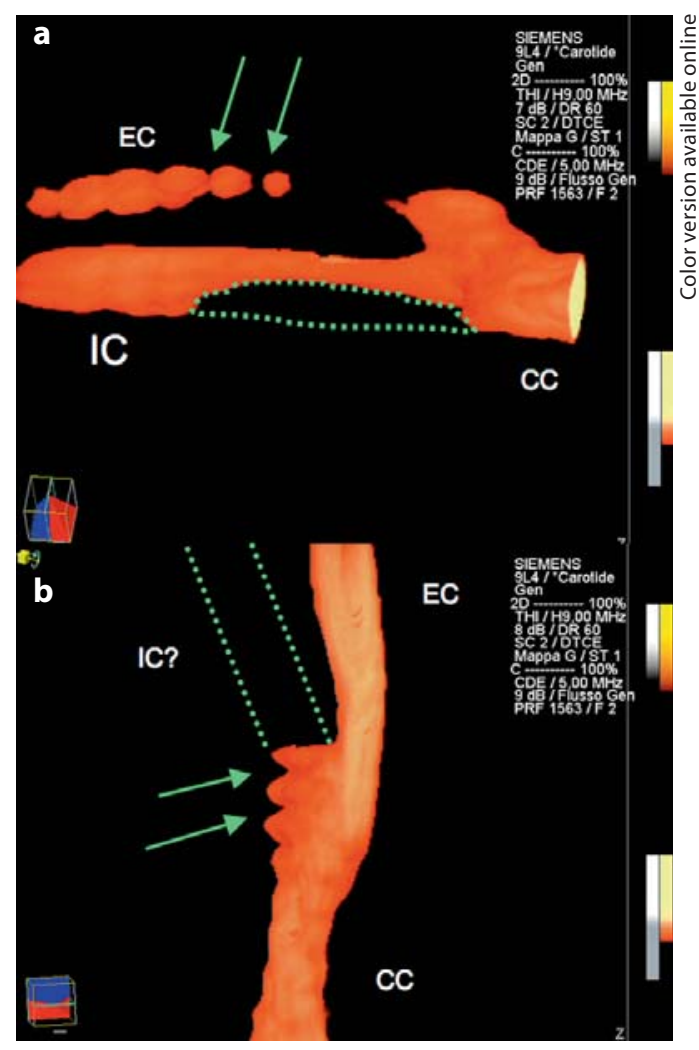




\section{Conclusions}

US is a powerful technique for visualizing carotid arteries, with the advantages of coupling the real-time morphological imaging with the study of the hemodynamic changes. 3D US is a feasible technique when performed by experienced examiners. It can improve carotid axis imaging, better presenting vessel course and caliber variations 'at a glance'. 3D US reconstructions from the inward flow can provide imaging of stenosis, but stenosis quantification must always take into account the assessment of plaque morphology and vessel wall.

\section{References}

1 De Castro S, Yao J, Pandian NG: Three-dimensional echocardiography: clinical relevance and application. Am J Cardiol 1998;18: 96G-102G.

-2 Badano LP, Dall'Armellina E, Monaghan MJ, Pepi M, Baldassi M, Cinello M, Fioretti PM: Real-time three-dimensional echocardiography: technological gadget or clinical tool? J Cardiovasc Med (Hagerstown) 2007; 8:144-162.

3 Mor-Avi V, Sugeng L, Lang RM: Three-dimensional adult echocardiography: where the hidden dimension helps. Curr Cardiol Rep 2008;10:218-225.

$\checkmark 4$ Correale M, Ieva R, Di Biase M: Real-time three-dimensional echocardiography: an update. Eur J Intern Med 2008;19:241-248.

$\checkmark 5$ Nesser HJ, Mor-Avi V, Gorissen W, Weinert L, Steringer-Mascherbauer R, Niel J, Sugeng L, Lang RM: Quantification of left ventricular volumes using three-dimensional echocardiographic speckle tracking: comparison with MRI. Eur Heart J 2009;30:1565-1573.

6 Shen O, Yagel S: The added value of 3D/4D ultrasound imaging in fetal cardiology: has the promise been fulfilled? Ultrasound $\mathrm{Ob}$ stet Gynecol 2010;35:260-262.

7 Dückelmann AM, Kalache KD: Three-dimensional ultrasound in evaluating the fetus. Prenat Diagn 2010;30:631-638.

8 Grigore M, Mare A: Applications of 3-D ultrasound in female infertility. Rev Med Chir Soc Med Nat Iasi 2009;113:1113-1119.

9 Zácková T, Järvelä IY, Tapanainen JS, Feyereisl J: Assessment of endometrial and ovarian characteristics using three dimensional power Doppler ultrasound to predict response in frozen embryo transfer cycles. Reprod Biol Endocrinol 2009;7:151.

10 Kalmantis K, Dimitrakakis C, Koumpis Ch, Tsigginou A, Papantoniou N, Mesogitis S, Antsaklis A: The contribution of three-dimensional power Doppler imaging in the preoperative assessment of breast tumors: a preliminary report. Obstet Gynecol Int 2009;2009:530-579.

- 11 Luo W, Numata K, Morimoto M, Nozaki A, Ueda M, Kondo M, Morita S, Tanaka K: Differentiation of focal liver lesions using threedimensional ultrasonography: retrospective and prospective studies. World J Gastroenterol 2010;16:2109-2119.
12 Espinoza J, Lee W, Comstock C, Romero R, Yeo L, Rizzo G, Paladini D, Viñals F, Achiron R, Gindes L, Abuhamad A, Sinkovskaya E, Russell E, Yagel S: Collaborative study on 4-dimensional echocardiography for the diagnosis of fetal heart defects: the COFEHD study. J Ultrasound Med 2010;29:1573-1580.

13 Hata T, Kanenishi K, Sasaki M: Four-dimensional sonographic assessment of fetal movement in the late first trimester. Int J Gynaecol Obstet. 2010;109:190-193.

14 Rustico MA, Mastromatteo C, Grigio M, Maggioni C, Gregori D, Nicolini U: Two-dimensional vs two- plus four-dimensional ultrasound in pregnancy and the effect on maternal emotional status: a randomized study. Ultrasound Obstet Gynecol 2005;25:468472.

$>15$ Lesage D, Angelini ED, Bloch I, Funka-Lea $\mathrm{G}$ : A review of $3 \mathrm{D}$ vessel lumen segmentation techniques: models, features and extraction schemes. Med Image Anal 2009;13:819-845.

-16 Janvier MA, Soulez G, Allard L, Cloutier G: Validation of 3D reconstructions of a mimicked femoral artery with an ultrasound imaging robotic system. Med Phys 2010;37: 3868-3879.

17 Sadat U, Teng Z, Young VE, Graves MJ, Gillard JH: Three-dimensional volumetric analysis of atherosclerotic plaques: a magnetic resonance imaging-based study of patients with moderate stenosis carotid artery disease. Int J Cardiovasc Imaging 2010;26: 897-904.

18 Rosenfield K, Boffetti P, Kaufman J, Weinstein R, Razvi S, Isner JM: Three-dimensional reconstruction of human carotid arteries from images obtained during noninvasive Bmode ultrasound examination. Am J Cardiol 1992;70:379-384.

19 Delcker A, Diener HC: Quantification of atherosclerotic plaques in carotid arteries by three-dimensional ultrasound. Br J Radiol 1994;67:672-678.

20 Fenster A, Downey DB, Cardinal HN: Threedimensional ultrasound imaging. Phys Med Biol 2001;46:67-99.

21 Landry A, Ainsworth C, Blake C, Spence JD, Fenster A: Manual planimetric measurement of carotid plaque volume using threedimensional ultrasound imaging. Med Phys 2007;34:1496-1505
22 Egger M, Spence JD, Fenster A, Parraga G: Validation of 3D ultrasound vessel wall volume: an imaging phenotype of carotid atherosclerosis. Ultrasound Med Biol 2007;33: 905-914.

23 Chiu B, Beletsky V, Spence JD, Parraga G, Fenster A: Analysis of carotid lumen surface morphology using three-dimensional ultrasound imaging. Phys Med Biol 2009;54: 1149-1167.

-24 Schminke U, Motsch L, Hilker L, Kessler C: Three-dimensional ultrasound observation of carotid artery plaque ulceration. Stroke 2000;31:1651-1655.

25 Pfister K, Rennert J, Greiner B, Jung W, Stehr A, Gössmann H, Menzel C, Zorger N, Prantl L, Feuerbach S, Kasprzak P, Jung EM: Presurgical evaluation of ICA-stenosis using 3D power Doppler, 3D color coded Doppler sonography, 3D B-flow and contrast enhanced B-flow in correlation to CTA/MRA: first clinical results. Clin Hemorheol Microcirc 2009;41:103-116.

-26 Mallett C, House AA, Spence JD, Fenster A, Parraga G: Longitudinal ultrasound evaluation of carotid atherosclerosis in one, two and three dimensions. Ultrasound Med Biol 2009;35:367-375.

27 Chiu B, Egger M, Spence JD, Parraga G, Fenster A: Quantification of carotid vessel wall and plaque thickness change using 3D ultrasound images. Med Phys 2008;35:3691-3710.

-28 Schminke U, Motsch L, Griewing B, Gaull M, Kessler C: Three-dimensional powermode ultrasound for quantification of the progression of carotid artery atherosclerosis. J Neurol 2000;247:106-111.

29 Krasinski A, Chiu B, Spence JD, Fenster A, Parraga G: Three-dimensional ultrasound quantification of intensive statin treatment of carotid atherosclerosis. Ultrasound Med Biol 2009;35:1763-1772.

-30 Barratt DC, Ariff BB, Humphries KN, Thom SA, Hughes AD: Reconstruction and quantification of the carotid artery bifurcation from 3-D ultrasound images. IEEE Trans Med Imaging 2004;23:567-583.

31 Vicenzini E, Ricciardi MC, Puccinelli F, Altieri M, Vanacore N, Di Piero V, Lenzi GL: Sonographic carotid plaque morphologic characteristics and vascular risk factors: results from a population study. J Ultrasound Med 2008;27:1313-1319. 
\$2 Vicenzini E, Giannoni MF, Puccinelli F, Ricciardi MC, Altieri M, Di Piero V, Gossetti B, Valentini FB, Lenzi GL: Detection of carotid adventitial vasa vasorum and plaque vascularization with ultrasound cadence contrast pulse sequencing technique and echo-contrast agent. Stroke 2007;38:2841-2843.

-33 Reneman RS, van Merode T, Hick P, Hoeks AP: Flow velocity patterns in and distensibility of the carotid artery bulb in subjects of various ages. Circulation 1985;71:500-509.

-34 Vicenzini E, Ricciardi MC, Sirimarco G, Di Piero V, Lenzi GL: Bilateral spontaneous internal carotid artery dissection with both early and very late recanalization: a case report. J Clini Ultrasound 2011;39:48-53.

>35 Mumoli N, Cei M: Asymptomatic carotid kinking. Circ J 2008;72:682-683.
36 Stanciulescu R, Ispas A, Filipoiu F, Bordei P, Galaman L, La Marca G, La Barbera G, Valentino F, Caruso G, Peri G, Valentino A, Lo Verde R, Valentino B: Anatomical variations of the carotid arteries: kinking, coiling, and tortuosity. Anatomical and functional considerations. IJAE 2010;115:161-168.

37 Togay-Işikay C, Kim J, Betterman K, Andrews C, Meads D, Tesh P, Tegeler C, Oztuna D: Carotid artery tortuosity, kinking, coiling: stroke risk factor, marker, or curiosity? Acta Neurol Belg 2005; 105:68-72.

38 La Barbera G, La Marca G, Martino A, Lo Verde R, Valentino F, Lipari D, Peri G, Cappello F, Valentino B: Kinking, coiling, and tortuosity of extracranial internal carotid artery: is it the effect of a metaplasia? Surg Radiol Anat 2006;28:573-580.

-39 Oliviero U, Scherillo G, Casaburi C, Di Martino M, Di Gianni A, Serpico R, Fazio S, Saccà $\mathrm{L}$ : Prospective evaluation of hypertensive patients with carotid kinking and coiling: an ultrasonographic 7-year study. Angiology 2003;54:169-175.
40 Faggioli G, Ferri M, Gargiulo M, Freyrie A, Fratesi F, Manzoli L, Stella A: Measurement and impact of proximal and distal tortuosity in carotid stenting procedures. J Vasc Surg 2007;46:1119-1124.

41 North American Symptomatic Carotid Endarterectomy Trialists' Collaborative Group: The final results of the NASCET trial. N Engl J Med 1998;339:1415-1425.

42 European Carotid Surgery Trialists' Collaborative Group: Randomised trial of endarterectomy for recently symptomatic carotid stenosis: final results of the MRC European Carotid Surgery Trial (ECST). Lancet 1998; 351:1379-1387. 